\title{
Diversity of angiogenesis among malignant bone tumors
}

\author{
TADAHIKO KUBO $^{1}$, SHOJI SHIMOSE $^{1}$, JUN FUJIMORI $^{1}$, KOJI ARIHIRO $^{2}$ and MITSUO OCHI $^{1}$ \\ ${ }^{1}$ Department of Orthopaedic Surgery, Graduate School of Biomedical Sciences, Hiroshima University; \\ ${ }^{2}$ Department of Anatomical Pathology, Hiroshima University Hospital, Minami-ku, Hiroshima 734-8551, Japan
}

Received May 29, 2012; Accepted July 11, 2012

DOI: $10.3892 / \mathrm{mco} .2012 .11$

\begin{abstract}
Several studies have demonstrated that angiogenesis assessed by microvessel density (MVD) correlates with patient prognosis in various types of cancer, whereas data regarding the relevance of angiogenesis and prognosis in malignant bone tumors are scarce and controversial. The aim of this study was to examine MVD in representative malignant bone tumors, such as osteosarcoma, chondrosarcoma and Ewing's sarcoma, in order to clarify the role of angiogenesis in prognosis. A total of 69 patients with malignant bone tumors, including 44 osteosarcomas, 20 chondrosarcomas and 5 Ewing's sarcomas, were reviewed retrospectively and treated at our hospital between 1980 and 2007. Biopsy or pre-chemotherapy surgical specimens were immunohistochemically stained with anti-CD34 antibody. The MVD values of osteosarcomas and Ewing's sarcomas were significantly higher compared to chondrosarcoma. In osteosarcomas with high MVD, American Joint Committee on Cancer stage IIA, good histological response to chemotherapy was significantly correlated with better diseasefree survival, while MVD was closely associated with age and chemotherapy response. In chondrosarcomas, the surgical margin (marginal and intralesional), MVD (high), tumor size $(\geq 8)$ and histological grade (grades 2 and 3) significantly correlated with a shorter disease-free survival, while MVD was closely associated with age and histological grade. These findings showed that osteosarcomas and Ewing's sarcomas were hypervascular, compared to chondrosarcomas. In osteosarcomas, hypervascularity induced good chemotherapy response, leading to better prognosis, while in chondrosarcomas, high MVD was associated with histological grade and predicted poor prognosis.
\end{abstract}

\section{Introduction}

Osteosarcoma is the most common primary malignant bone tumor, characterized by high metastatic potential.

Correspondence to: Dr Tadahiko Kubo, Department of Orthopaedic Surgery, Graduate School of Biomedical Sciences, Hiroshima University, 1-2-3 Kasumi, Minami-ku, Hiroshima 734-8551, Japan E-mail: kubot@hiroshima-u.ac.jp

Key words: angiogenesis, osteosarcoma, chondrosarcoma, Ewing's sarcoma, prognosis
Chemotherapy protocols, commencing in the mid-1970s, have increased during the 5-year disease-free survival rate from approximately $15-20$ to $60-70 \%(1,2)$. Ewing's sarcoma is the second most common malignant bone tumor in children. The inclusion of cytotoxic polychemotherapy into multimodal treatment strategies has led to marked prognostic improvements in patients with Ewing's sarcoma with 5-year survival rates reaching $75 \%$ for non-metastasized and $20-40 \%$ for primary metastasized Ewing's sarcoma $(3,4)$. Over the last two decades, however, attempts to further intensify therapy using conventional chemotherapeutic drugs, including highdose methotrexate, doxorubicin, cisplatin and high-dose ifosfamide, have not delivered significantly improved results. Furthermore, despite aggressive surgical and chemotherapy approaches, patients with unresectable primary tumors or with clinically evident metastases still had a poor prognosis. Thus, for patients with refractory osteosarcoma and Ewing's sarcoma more beneficial prognostic factors and more effective therapeutic modalities are needed.

Chondrosarcoma is the most common primary bone sarcoma in adulthood, generally resistant to radiotherapy and chemotherapy, thus surgical wide resection is the only curative treatment. The rates of local recurrence, distant metastasis and survival vary, while the histological grade of cartilage tumors is currently considered to be the best tool for the assessment of prognosis (5). However, the histological determination of the tumor grade is basically subjective and pathologists do not always agree on the extent or level of cytological factors of chondrosarcoma cells (6). It is often difficult to distinguish a low-grade chondrosarcoma and benign processes, such as enchondroma and osteochondroma. To assess prognosis, more complex objective methods have been sought.

Tumor growth and metastasis have been shown to strongly depend on angiogenesis. Folkman et al (7) demonstrated the mechanisms and relevance of angiogenesis in malignant tumors. Since then, several reports demonstrated that angiogenesis assessed by microvessel density (MVD) was correlated with patient prognosis in different types of cancer (8), whereas data regarding the relevance of angiogenesis and prognosis in malignant bone tumors remain scarce and controversial. The aim of this study was to examine MVD in representative malignant bone tumors, such as osteosarcoma, chondrosarcoma and Ewing's sarcoma, with a view to clarify the role of angiogenesis in prognosis and refer to therapeutic strategies. 


\section{Patients and methods}

Patients. A total of 69 patients with malignant bone tumors, including 44 osteosarcomas, 20 chondrosarcomas and 5 Ewing's sarcomas were reviewed retrospectively and treated at our hospital between 1980 and 2007. Subsequent to approval of the present study by the Institutional Review Board, clinical data from the patients' medical charts were reviewed. Biopsy or pre-chemotherapy surgical specimens were immunohistochemically stained.

Surgical resection specimens were used to evaluate surgical margins and histologic response to pre-operative chemotherapy. The surgical margins were classified using the method described by Kawaguchi et al (9), whereby surgical margins are evaluated according to the distance between the tumor's margin and reactive zone, and divided into four categories: curative, wide, marginal and intralesional. The reactive zone is composed of hemorrhagic tissue, scar tissue, degenerated muscle, edema or the tumor capsule. If the surgical margin is $>5 \mathrm{~cm}$ outside the reactive zone, the margin is graded as curative, while it is referred to as wide if the margin is $<5 \mathrm{~cm}$ and outside the reactive zone. A margin in the reactive zone is graded as marginal, while one passing through a tumor as intralesional. Histologic necrosis subsequent to pre-operative chemotherapy was determined according to the Huvos grading system as described previously (10). Each case was defined as good (Huvos grades III and IV) or poor responders (grades I and II).

Immunohistochemical staining. Each tissue block was cut into 6- $\mu \mathrm{m}$ sections, transferred to MAS-coated glass slides (Matsunami, Osaka, Japan), deparaffinized in xylene, rehydrated in a graded series of decreasing ethanol concentrations, then rinsed in TBST $(50 \mathrm{mM}$ Tris- $\mathrm{HCl}, \mathrm{pH} 7.6$, containing $0.3 \mathrm{M}$ sodium chloride and $0.1 \%$ Tween-20). Tissue sections were immersed in Target Retrieval Solution (DakoCytomation Inc., Carpinteria, CA, USA), and subjected to a hot water-bath for $20 \mathrm{~min}$. Subsequent to antigen retrieval, a cooling period of 20 min followed. CD34 antibody (Ready-to use N-series Qbend 10; DakoCytomation, Inc.) was incubated with the tissue sections for $30 \mathrm{~min}$ at room temperature in a moisture chamber, then the polymer-peroxidase method was used (EnVision ${ }^{\circledR}+$ /HRP; DakoCytomation, Inc.). The reaction products were visualized by exposing the sections to 3,3'-diaminobenzidine. Nuclei were lightly counterstained for $\sim 10 \mathrm{sec}$ with Gill's formulation \#2 hematoxylin. Non-specific reactivity was assessed by omitting the primary antibody.

Microvessel counting. MVD values were calculated by two independent observers, according to Weidner's method (11). Briefly, in each tumor, hot-spot areas exhibiting the highest vessel density were identified by scanning tumor sections at low-power magnification (x40). The maximum vessel density was determined from these hot-spot areas at fields under highpower magnification (x400, HPF), and the mean of the counts for the three fields was calculated. For a structure to be defined a vessel, vessel lumens were not necessary. MVD was classified as high when $\geq 39.7,12.9$ and 44.6 (the mean value)/HPF in osteosarcomas, chondrosarcomas and Ewing's sarcomas, respectively.
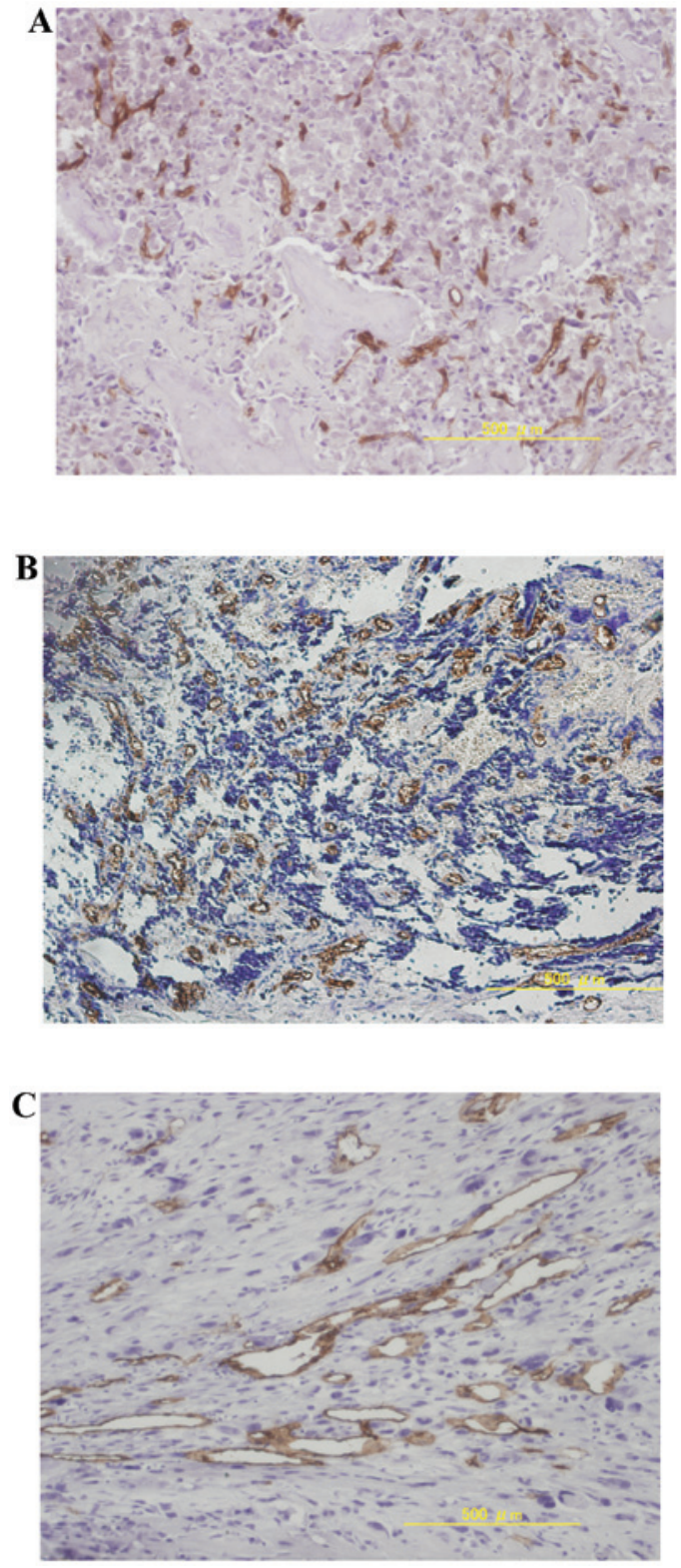

Figure 1. Representative immunohistochemical staining of high MVD in (A) osteosarcoma, (B) chondrosarcoma and (C) Ewing's sarcoma. Original magnification, x100. MVD, microvessel density.

Statistical analysis. Disease-free survival was defined as the interval from diagnosis to relapse or the last follow-up. All survival analyses were evaluated with the Kaplan-Meier method and the log-rank test. Statistical significance of MVD among osteosarcomas, chondrosarcomas and Ewing's sarcomas was determined by the Student's t-test. The association of MVD with clinicopathological parameters was analyzed, using the Student's t-test. The analyses were carried out with Excel Statistics 2008 (Social Survey Research Information, Co., Ltd., Tokyo, Japan). $\mathrm{P}<0.05$ was considered to indicate a statistically significant difference.

\section{Results}

Diversity of angiogenesis in malignant bone tumors. The mean \pm standard deviation of MVD was $39.7 \pm 31.8,12.9 \pm 32.2$, and $44.6 \pm 33.8$ in osteosarcomas, chondrosarcomas and 
Table I. Prognostic variables in osteosarcoma as determined by univariate analysis of disease-free survival.

\begin{tabular}{|c|c|c|c|}
\hline Variable & $\begin{array}{c}5 \text {-year DFS } \\
(\mathrm{n}=44), \%\end{array}$ & $95 \% \mathrm{CI}$ & P-value ${ }^{a}$ \\
\hline \multicolumn{4}{|l|}{ Age (years) } \\
\hline$<20$ & 56 & $40-72$ & 0.203 \\
\hline$\geq 20$ & 38 & $0-94$ & \\
\hline \multicolumn{4}{|l|}{ Gender } \\
\hline Male & 60 & $40-80$ & 0.697 \\
\hline Female & 49 & $27-71$ & \\
\hline \multicolumn{4}{|l|}{ Tumor site } \\
\hline Femur & 55 & $21-89$ & 0.944 \\
\hline Tibia or fibula & 62 & $43-80$ & \\
\hline \multicolumn{4}{|l|}{ Surgical stage } \\
\hline IIA & 89 & $68-100$ & 0.035 \\
\hline IIB & 47 & $26-68$ & \\
\hline \multicolumn{4}{|l|}{ Histology } \\
\hline Osteoblastic & 52 & $32-71$ & 0.758 \\
\hline Fibroblastic & 60 & $17-100$ & \\
\hline \multicolumn{4}{|l|}{ Surgery } \\
\hline Amputation & 47 & $23-71$ & 0.529 \\
\hline Limb salvage & 60 & $40-79$ & \\
\hline \multicolumn{4}{|c|}{ Response to chemotherapy } \\
\hline Poor & 40 & $20-61$ & 0.026 \\
\hline Good & 76 & $53-100$ & \\
\hline \multicolumn{4}{|l|}{ MVD } \\
\hline Low & 38 & $19-56$ & 0.009 \\
\hline High & 87 & $69-100$ & \\
\hline
\end{tabular}

${ }^{a}$ Log-rank test was used for survival analyses. DFS, disease-free survival; CI, confidence interval; MVD, microvessel density.

Ewing's sarcomas, respectively. The MVD values of osteosarcomas and Ewing's sarcomas were significantly higher compared to chondrosarcomas $(\mathrm{P}<0.05$, Student's t-test $)$. Representative immunohistochemical staining of high MVD in osteosarcomas, chondrosarcomas and Ewing's sarcomas is shown in Fig. 1.

Survival analysis in osteosarcomas. A total of 42 high-grade osteosarcomas and 2 low-grade osteosarcomas were reviewed. The median age at the time of surgery was 21 years (range, 10-88). There were 20 males and 24 females. The tumor sites were the pelvis in 2 patients and the extremities in 42 patients ( 27 femurs, 10 tibias or fibulas and 5 humeri). According to the 6th edition of the American Joint Committee on Cancer (AJCC) Staging Manual, 2 tumors were stage IB, 10 were stage IIA, 23 were stage IIB and 2 were stage IV (7 missing in tumor size). Between 1980 and 1997, 22 patients received conventional chemotherapy, including high-dose methotrexate, doxorubicin and cisplatin. The combinations of such agents were made empirically. Since 1997, two established chemotherapy regimens have been in use. NECO-95J and
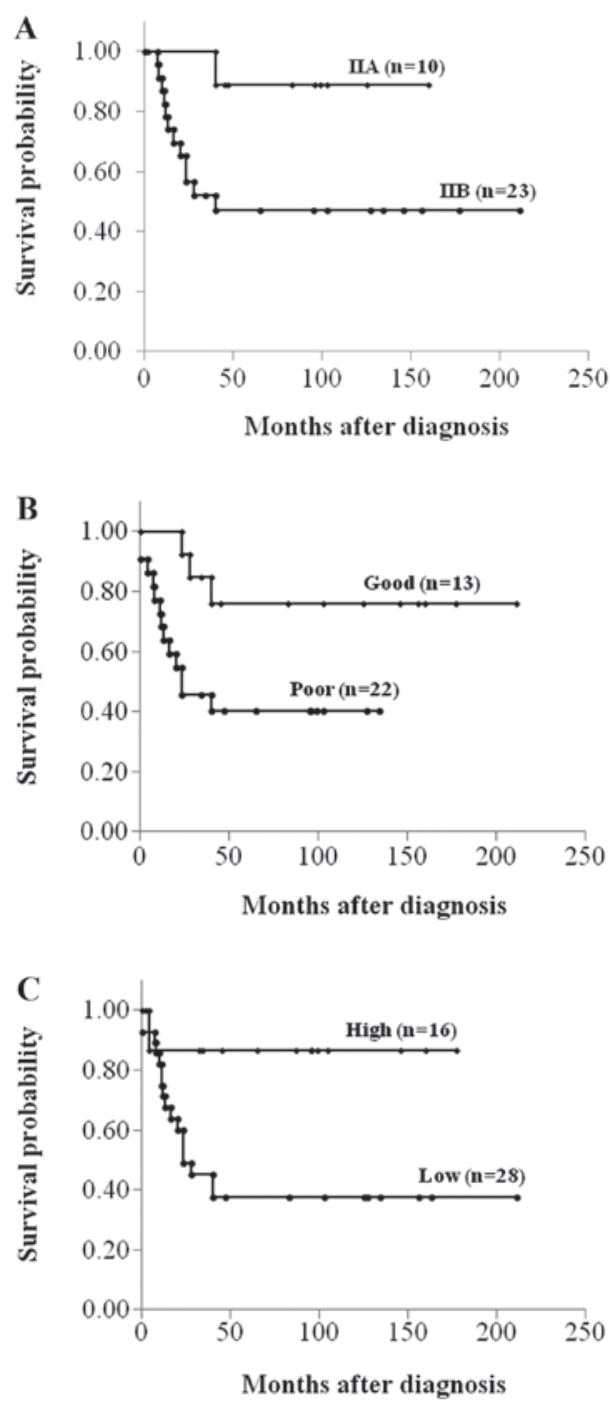

Figure 2. Kaplan-Meier plots of disease-free survival for osteosarcoma patients, according to (A) the American Joint Committee on Cancer surgical stage, (B) histological response to chemotherapy and (C) MVD.

$\mathrm{K} 2$ protocols were applied in the case of 12 patients between 1997 and 2002 and 8 patients between 2002 and 2006, respectively. Two patients ( $>70$ years-old) received no chemotherapy $(12,13)$. The operative treatments consisted of $26 \mathrm{limb}$ salvage operations and 18 ablations. The surgical margins of all limb salvage operations were wide, while those of ablations were wide or curative. Histologic subtypes comprised osteoblastic in 28 tumors, fibroblastic in 6, chondroblastic in 3 and others in 7. There were 13 good and 22 poor responders to preoperative chemotherapy (7 missing). The median follow-up was 5 years and 11 months (range, 2-211 months). There was 1 local recurrence and there were 19 distant metastases during patients' clinical courses. Fifteen patients were deceased, 27 demonstrated no evidence of disease, while 2 were alive with signs of disease. The 5-year actual disease-free survival rate was $54.6 \%$.

Sixteen of 42 (38.1\%) osteosarcomas demonstrated high MVD. High MVD, AJCC stage IIA and good histological response to chemotherapy correlated significantly with better disease-free survival $(\mathrm{P}<0.05, \log$-rank test) (Fig. 2 and Table I). There were statistically significant associations of 
Table II. Association of MVD with clinicopathological characteristics in 44 patients with osteosarcoma.

\begin{tabular}{lcc}
\hline Variable & MVD & P-value \\
\hline Age (years) & & \\
$<20$ & $46.4 \pm 33.7$ & 0.007 \\
$\geq 20$ & $19.6 \pm 10.2$ & \\
Gender & & \\
$\quad$ Male & $42.6 \pm 33.8$ & 0.293 \\
Female & $37.2 \pm 30.4$ & \\
Tumor site & & \\
Femur & $42.0 \pm 32.0$ & 0.309 \\
Tibia or fibula & $36.1 \pm 30.9$ & \\
Surgical stage & & \\
IIA & $44.1 \pm 25.5$ & 0.226 \\
IIB & $35.2 \pm 32.6$ & \\
Histology & & \\
Osteoblastic & $40.2 \pm 32.5$ & 0.255 \\
Fibroblastic & $31.0 \pm 20.8$ & \\
Surgery & & \\
Amputation & $35.0 \pm 32.9$ & 0.213 \\
Limb salvage & $42.9 \pm 31.2$ & \\
Response to chemotherapy & & \\
Poor & $29.6 \pm 26.5$ & 0.043 \\
Good & $48.3 \pm 35.7$ & \\
a Student's t-test was used to evaluate the association between MVD \\
and parameters. MVD, microvessel density. \\
\hline
\end{tabular}

MVD with age and chemotherapy response $(\mathrm{P}<0.05$, Student's t-test) (Table II).

Survival analysis in chondrosarcomas. The patients with chondrosarcoma presented primary lesions without any metastases. The median age at the time of surgery was 42 years (range, 15-71). There were 8 males and 12 females. The tumor sites were 12 in the trunk ( 9 pelvises, 1 rib, 1 scapula and 1 vertebra) and 8 in the extremity (4 tibias, 3 humeri and 1 femur). The median tumor size, defined as the maximum diameter of the tumor at pathological analysis, was $11.7 \mathrm{~cm}$ (range, 4-27). Histological grades widely used were 14 in grade 1, 3 in grade 2, and 3 in grade 3 (including one de-differentiated chondrosarcoma and one mesenchymal chondrosarcoma). Sixteen patients were treated with wide, 2 patients with marginal and 2 patients with intralesional excision. The median follow-up period was 6 years and 6 months (range, 1-192 months). There was 1 local recurrence and there were 3 lung metastases during the patients' clinical courses. One patient was deceased due to the disease, 1 of another cause, while 17 showed no evidence of disease and 1 was alive with signs of the disease. The 5-year actual disease-free survival rate was $81.3 \%$.

Four of 20 (20\%) chondrosarcomas showed high MVD. Surgical margin (marginal and intralesional), MVD (high),
Table III. Prognostic variables in chondrosarcoma as determined by univariate analysis of disease-free survival.

\begin{tabular}{|c|c|c|c|}
\hline Variable & $\begin{array}{c}5 \text {-year DFS } \\
(\mathrm{n}=20), \%\end{array}$ & $95 \% \mathrm{CI}$ & P-value \\
\hline \multicolumn{4}{|l|}{ Age (years) } \\
\hline$<40$ & 71 & $38-100$ & 0.468 \\
\hline$\geq 40$ & 89 & $68-100$ & \\
\hline \multicolumn{4}{|l|}{ Gender } \\
\hline Male & 70 & $34-100$ & 0.216 \\
\hline Female & 89 & $68-100$ & \\
\hline \multicolumn{4}{|l|}{ Tumor size $(\mathrm{cm})$} \\
\hline$<8$ & 100 & $0-100$ & 0.020 \\
\hline$\geq 8$ & 66 & $34-98$ & \\
\hline \multicolumn{4}{|l|}{ Tumor site } \\
\hline Extremity & 100 & $0-100$ & 0.861 \\
\hline Trunk & 66 & $34-98$ & \\
\hline \multicolumn{4}{|l|}{ Histological grade } \\
\hline 1 & 93 & $79-100$ & 0.033 \\
\hline 2 and 3 & 50 & $1-99$ & \\
\hline \multicolumn{4}{|l|}{ Surgical margin } \\
\hline Wide & 84 & $64-100$ & 0.017 \\
\hline $\begin{array}{l}\text { Intralesional and } \\
\text { marginal }\end{array}$ & 75 & $33-100$ & \\
\hline \multicolumn{4}{|l|}{ MVD } \\
\hline Low & 82 & $68-100$ & 0.025 \\
\hline High & 67 & $13-100$ & \\
\hline
\end{tabular}

${ }^{a}$ Log-rank test was used for survival analyses. DFS, disease-free survival; CI, confidence interval; MVD, microvessel density.

tumor size $(\geq 8)$ and histological grade (grades 2 and 3) correlated significantly with a shorter disease-free survival $(\mathrm{P}<0.05$, log-rank test) (Table III). There were statistically significant associations of MVD with age and histological grade $(\mathrm{P}<0.05$, Student's t-test) (Table IV).

\section{Discussion}

In this study, the diversity of angiogenesis in malignant bone tumors was demonstrated. Osteosarcomas and Ewing's sarcomas were hypervascular, compared to the chondrosarcomas. Although MVD in osteosarcomas and chondrosarcomas was a possible prognostic marker, it had reverse effects on prognosis in osteosarcomas and chondrosarcomas. High MVD correlated significantly with a higher disease-free survival in osteosarcomas, and a lower disease-free survival in chondrosarcomas.

Several studies have examined the clinical significance of angiogenesis-related biomarkers in osteosarcomas. Most of them, however, were studies with small series, thus the role of angiogenesis remains to be clarified. Mikulić et al (14) demonstrated that high MVD was predictive of a greater probability of distant metastasis and of shorter overall and disease-free 
Table IV. Association of MVD with clinicopathological characteristics in 20 patients with chondrosarcoma.

\begin{tabular}{lcc}
\hline Variable & MVD & P-value $^{\mathrm{a}}$ \\
\hline $\begin{array}{l}\text { Age (years) } \\
<40\end{array}$ & $30.1 \pm 52.0$ & 0.039 \\
$\geq 40$ & $3.6 \pm 5.1$ & \\
Gender & & \\
$\quad$ Male & $14.6 \pm 30.6$ & 0.432 \\
Female & $11.9 \pm 34.3$ & \\
$\quad$ Tumor size (cm) & & \\
$<8$ & $1.9 \pm 3.0$ & 0.087 \\
$\geq 8$ & $21.9 \pm 42.1$ & \\
$\quad \begin{array}{l}\text { Tumor site } \\
\text { Extremity }\end{array}$ & $11.4 \pm 27.3$ & 0.428 \\
$\quad$ Trunk & $14.1 \pm 37.1$ & \\
Histological grade & & \\
1 & $1.7 \pm 2.5$ & 0.015 \\
2 and 3 & $33.6 \pm 50.0$ & \\
Surgical margin & & \\
Wide & & \\
Intralesional and marginal & $6.6 \pm 6.4$ & 0.337 \\
\hline
\end{tabular}

aStudent's t-test was used to evaluate the association between MVD and parameters. MVD, microvessel density.

survival. Kaya et al (15) described vascular endothelial growth factor (VEGF), a well-known proangiogenic factor. Patients with a VEGF-positive tumor showed a shorter disease-free, as well as an overall survival period. Numerous other reports, however, failed to find a correlation between MVD or VEGF and survival (16-18). Conversely, in their study Kreuter et al examined 60 patients with high-grade central osteosarcoma and found, that patients with a high MVD had significantly longer overall and relapse-free survival period, compared to patients with low MVD (19). A good response to chemotherapy was significantly correlated with a higher MVD, to some extent possibly due to the improved accessibility of chemotherapy to proliferating osteosarcoma cells. Tumor microcirculation is an important factor in drug delivery to cancer cells. The efficacy of drug delivery is much higher in a tumor with a high degree of microvessels compared to a tumor with low MVD, especially in a chemotherapy-sensitive tumor, such as osteosarcoma. In contrast to other studies, patients in the present study received a standardized treatment with intensive pre- and post-operative chemotherapy, as well as intensive chemotherapy, delivering results consistent with Kreuter's results, suggesting that hypervascularity might induce good response to chemotherapy, thus leading to better prognosis. The improvement of the distribution of hypovascular osteosarcoma is required.

Angiogenesis is one of the probable elements involved in the transformation and progression of neoplasia. It is particularly significant in the progression of cartilage neoplasia, since cartilage neoplasia has some phenotypic links to cartilage, a vascular tissue. Several studies have demonstrated that cartilage tumors have microvessels in the development and progression, while the amount of vascularity correlates with tumor grade (20-22). To further elucidate angiogenesis in cartilage tumors, Kalinski et al (23) used double-labeling immunohistochemistry, using von Willebrad factor and MIB-1 aiming to show that the proliferating capillary index, better characterizing the angiogenic properties of a distinct tumor compared to MVD, was significantly higher in conventional chondrosarcoma grades II and III compared to enchondroma, chondrosarcoma grade I or de-differentiated chondrosarcoma. Nakagawa et al (24) demonstrated that there was a significant association of CD34 with histological grades, although not with overall prognosis. In the present study, high MVD was associated with histological grade and predicted poor prognosis in chondrosarcoma. Surgical treatment with wide resection was the mainstay of therapy for chondrosarcoma, whereas chemotherapy and radiotherapy were negligible in their treatment. At present, there is no widely adopted adjuvant therapy (25). Results drawn from this study seem to be of value for the development of antiangiogenic chemotherapy, for patients with chondrosarcoma.

MVD was significantly associated with age in both osteosarcomas and chondrosarcomas and was higher in patients aged $<20$ years with osteosarcoma and patients aged $<40$ years with chondrosarcoma. These interesting findings have never been described before, however, the present study was limited to a small sample size and the multivariate model did not converge. Therefore, to confirm the conclusion, additional investigations involving a larger number of patients is required.

In conclusion, the present study showed that there was diversity of angiogenesis in malignant bone tumors. Osteosarcomas and Ewing's sarcomas were hypervascular, compared to chondrosarcoma. In osteosarcomas, hypervascularity induced good chemotherapy response, leading to better prognosis, while in hypovascular osteosarcoma a better drug distribution in tumors is needed. In chondrosarcomas, high MVD was associated with histological grade and predicted poor prognosis, suggesting the development of antiangiogenic chemotherapy for patients with chondrosarcoma. These encouraging data should lead to additional studies involving larger sample size, to exclude the possibility that these divergences are caused by differences in patients' characteristics and methodology in staining and analysis.

\section{References}

1. Bacci G, Briccoli A, Ferrari S, et al: Neoadjuvant chemotherapy for osteosarcoma of the extremity: long-term results of the Rizzoli's 4th protocol. Eur J Cancer 37: 2030-2039, 2001.

2. Bielack SS, Kempf-Bielack B, Delling G, et al: Prognostic factors in high-grade osteosarcoma of the extremities or trunk: an analysis of 1,702 patients treated on neoadjuvant cooperative osteosarcoma study group protocols. J Clin Oncol 20: 776-790, 2002.

3. Paulussen M, Fröhlich B and Jürgens H: Ewing tumour: incidence, prognosis and treatment options. Paediatr Drugs 3: 899-913, 2001

4. Paulussen M, Ahrens S, Dunst J, et al: Localized Ewing tumor of bone: final results of the cooperative Ewing's Sarcoma Study CESS 86. J Clin Oncol 19: 1818-1829, 2001.

5. Rizzo M, Ghert MA, Harrelson JM and Scully SP: Chondrosarcoma of bone: analysis of 108 cases and evaluation for predictors of outcome. Clin Orthop Relat Res 391: 224-233, 2001. 
6. Welkerling H, Kratz S, Ewerbeck V and Delling G: A reproducible and simple grading system for classical chondrosarcomas Analysis of 35 chondrosarcomas and 16 enchondromas with emphasis on recurrence rate and radiological and clinical data. Virchows Arch 443: 725-733, 2003.

7. Folkman J, Watson K, Ingber D and Hanahan D: Induction of angiogenesis during the transition from hyperplasia to neoplasia. Nature 339: 58-61, 1989.

8. Hasan J, Byers R and Jayson GC: Intra-tumoural microvessel density in human solid tumours. Br J Cancer 86: 1566-1577, 2002.

9. Kawaguchi N, Matumoto S and Manabe J: New method of evaluating the surgical margin and safety margin for musculoskeletal sarcoma, analysed on the basis of 457 surgical cases. J Cancer Res Clin Oncol 121: 555-563, 1995.

10. Huvos AG (ed): Bone Tumors: Diagnosis, Treatment, and Prognosis. 2nd edition. W.B. Saunders, Philadelphia, pp85-155, 1991.

11. Weidner N, Semple JP, Welch WR and Folkman J: Tumor angiogenesis and metastasis-correlation in invasive breast carcinoma. N Engl J Med 324: 1-8, 1991.

12. Iwamoto $\mathrm{Y}$, Tanaka K, Isu K, et al: Multiinstitutional phase II study of neoadjuvant chemotherapy for osteosarcoma (NECO study) in Japan: NECO-93J and NECO-95J. J Orthop Sci 14: 397-404, 2009.

13. Kimura H, Tsuchiya H, Shirai T, et al: Caffeine-potentiated chemotherapy for metastatic osteosarcoma. J Orthop Sci 14: $556-565,2009$

14. Mikulić D, Ilić I, Cepulić M, et al: Tumor angiogenesis and outcome in osteosarcoma. Pediatr Hematol Oncol 21: 611-619, 2004.

15. Kaya M, Wada T, Nagoya S, Sasaki M, Matsumura T and Yamashita T: The level of vascular endothelial growth factor as a predictor of a poor prognosis in osteosarcoma. J Bone Joint Surg Br 91: 784-788, 2009.
16. Mantadakis E, Kim G, Reisch J, et al: Lack of prognostic significance of intratumoral angiogenesis in nonmetastatic osteosarcoma. J Pediatr Hematol Oncol 23: 286-289, 2001.

17. Ek ET, Ojaimi J, Kitagawa Y and Choong PF: Does the degree of intratumoural microvessel density and VEGF expression have prognostic significance in osteosarcoma? Oncol Rep 16: 17-23, 2006.

18. Ek ET, Ojaimi J, Kitagawa Y and Choong PF: Outcome of patients with osteosarcoma over 40 years of age: is angiogenesis a marker of survival? Int Semin Surg Oncol 3: 7, 2006.

19. Kreuter M, Bieker R, Bielack SS, et al: Prognostic relevance of increased angiogenesis in osteosarcoma. Clin Cancer Res 10: 8531-8537, 2004

20. Ayala G, Liu C, Nicosia R, Horowitz S and Lackman R: Microvasculature and VEGF expression in cartilaginous tumors. Hum Pathol 31: 341-346, 2000.

21. McGough RL, Aswad BI and Terek RM: Pathologic neovascularization in cartilage tumors. Clin Orthop Relat Res 397: 76-82, 2002.

22. McGough RL, Lin C, Meitner P, Aswad BI and Terek RM: Angiogenic cytokines in cartilage tumors. Clin Orthop Relat Res 397: 62-69, 2002.

23. Kalinski T, Sel S, Kouznetsova I, Röpke M and Roessner A: Heterogeneity of angiogenesis and blood vessel maturation in cartilage tumors. Pathol Res Pract 205: 339-345, 2009.

24. Nakagawa SA, Lopes A, Lopes de Carvalho A, et al: Nitric oxide synthases, cyclooxygenase-2, nitrotyrosine, and angiogenesis in chondrosarcoma and their relation to prognosis. J Bone Joint Surg Am 92: 1738-1746, 2010.

25. Riedel RF, Larrier N, Dodd L, Kirsch D, Martinez S and Brigman BE: The clinical management of chondrosarcoma. Curr Treat Options Oncol 10: 94-106, 2009. 